\title{
Thresholds and tolerance of physical pain among young adults who self-injure
}

\author{
Katrina McCoy MS, William Fremouw PhD, Daniel W McNeil PhD
}

\begin{abstract}
K McCoy, W Fremouw, DW McNeil. Thresholds and tolerance of physical pain among young adults who self-injure. Pain Res Manage 2010;15(6):371-377.

Prevalence rates of nonsuicidal self-injury among college students range from $17 \%$ to $38 \%$. Research indicates that individuals with borderline personality disorder who self-injure sometimes report an absence of pain during self-injury. Furthermore, self-injury in the absence of pain has been associated with more frequent suicide attempts. The present study examined pain thresholds and tolerance among 44 college students (11 who engaged in self-injury and 33 who did not). Pain thresholds and tolerance were measured using an algometer pressure device that was used to produce pain in previous laboratory research. Participants who engaged in selfinjury had a higher pain tolerance than those who did not. In addition, participants who engaged in self-injury rated the pain as less intense than participants who did not. ANCOVAs revealed that depression was associated with pain rating and pain tolerance.
\end{abstract}

Key Words: Pain perception; Pain tolerance; Self-harm; Self-injury

\section{Les seuils de douleur physique et la tolérance à cette douleur chez les jeunes adultes qui s'automutilent}

Les taux de prévalence d'automutilation non suicidaire chez les étudiants universitaires oscillent entre $17 \%$ et $38 \%$. Selon les recherches, les personnes ayant des troubles de la personnalité limites qui s'automutilent déclarent parfois ne pas ressentir la douleur pendant l'automutilation. Par ailleurs, l'automutilation en l'absence de douleur s'associe à des tentatives de suicide plus fréquentes. La présente étude visait à évaluer les seuils de douleur et la tolérance à la douleur chez 44 étudiants universitaires (11 qui s'automutilaient et 33 qui ne s'automutilaient pas). Les chercheurs ont mesuré les seuils de douleur et la tolérance à la douleur au moyen d'un algomètre utilisé pour produire de la douleur dans le cadre de recherches de laboratoire antérieures. Les participants qui s'automutilaient toléraient mieux la douleur que ceux qui ne s'automutilaient pas. De plus, ils classaient la douleur comme moins intense que ceux qui ne s'automutilaient pas. L'analyse de covariance a révélé que la dépression s'associait au classement de la douleur et à la tolérance à la douleur.
Self-injury is defined as the intentional destruction of body tissue in the absence of a desire for death that is not culturally or socially sanctioned. Behaviours commonly included under the rubric of self-injury are cutting, burning and scratching (1). Tattoos and body piercing are culturally sanctioned and are, therefore, not typically described as self-injury. Other terms used to refer to self-injury include deliberate self-injury, deliberate self-harm and self-mutilation (1-4). The term nonsuicidal self-injury (NSSI) is increasingly used to distinguish between self-injury intended to cause death and self-injury not intended to cause death (1). NSSI is an increasingly prevalent public health problem. The prevalence of NSSI has been estimated at $4 \%$ among the general adult population (5) and approximately $14 \%$ among adolescents (6). Prevalence rates of NSSI are even higher among college students, ranging from $17 \%$ to $38 \%(7,8)$.

NSSI is associated with psychological distress among nonclinical populations. Among young adults, NSSI has been associated with a history of suicide attempts, suicidal ideation, having a plan for suicide and suicidal gestures (8). Whitlock et al (8) found that $75.9 \%$ of a sample of college students who had a history of NSSI had considered or attempted suicide. Research $(7,9-11)$ has consistently demonstrated that
NSSI is associated with mental health problems including major depression and anxiety, feelings of hopelessness, and dissociation. Klonsky et al (11) found that military recruits with a history of NSSI reported more symptoms of anxiety and depression than individuals without a history of NSSI. Similar findings emerged among adolescents (6). Gratz et al (7) found a significant correlation $(0.33)$ between dissociation as measured by self-report with the Dissociative Experiences Scale (DES) and frequency of NSSI among a sample of college students. NSSI is also associated with several personality disorders, anorexia nervosa, substance disorders, eating disorders and posttraumatic stress disorder (9).

One issue that is not well understood is the degree to which individuals who self-injure experience pain during NSSI. The literature suggests that as many as $60 \%$ of individuals with borderline personality disorder (BPD) and $80 \%$ of adolescent psychiatric inpatients report little or no pain during NSSI (4,12-14). Joiner (15) argued that greater experience with selfinjury leads to an increased capacity for lethal self-harm through the desensitization to fear and pain by which it may be accompanied. Consistent with this theory, Nock et al (16) found that adolescent inpatients who reported the absence of pain during NSSI reported twice as many suicide attempts as

Department of Psychology, West Virginia University, Morgantown, West Virginia, USA

Correspondence: Ms Katrina McCoy, Department of Psychology, West Virginia University, 53 Campus Drive, Box 6040, Morgantown,

West Virginia 26506-6040, USA. Telephone 304-293-2001, e-mail Katrina.McCoy@mail.wvu.edu 
those who reported experiencing pain during NSSI. Russ et al (17) found that individuals with BPD who reported the absence of pain during NSSI reported significantly reduced depression, anxiety, anger and confusion subsequent to a laboratory pain task (the cold pressor test), suggesting that the absence of pain during NSSI may be associated with the affect regulation function of NSSI. This is alarming in light of a growing body of literature suggesting that individuals who engage in NSSI with intent to alleviate negative affect are more likely to feel hopeless and have a history of medically severe suicide attempts compared with individuals who engage in NSSI for other reasons $(4,18)$.

Despite the interest in pain perception among inpatients and individuals with BPD who self-injure, few studies have examined differences in pain perception among individuals who self-injure compared with those who do not. Furthermore, the aforementioned studies relied on participants' self-report of pain perception during past episodes of NSSI or during a laboratory pain task. To date, only one study (12) has corroborated self-report of pain perception among individuals who self-injure with a behavioural measure of pain tolerance and threshold. Using a tourniquet pain test, Bohus et al (12) found that individuals with BPD and a history of NSSI have a significantly higher threshold and tolerance for pain than a control group of nonclinical participants.

The aforementioned literature suggests that individuals who self-injure may have a higher pain tolerance and threshold than individuals who do not self-injure, but has focused exclusively on inpatient samples or individuals with BPD. This hypothesis has not been tested among community samples despite the high rates of NSSI among college populations. The purpose of the current study was to examine pain threshold and tolerance in a sample of college students with a history of NSSI compared with controls to determine whether previous findings can be generalized from the inpatient and BPD population to a noninpatient sample. It was hypothesized that participants who self-injure would have a higher pain tolerance and threshold, and lower pain ratings than those who do not self-injure. Furthermore, it was hypothesized that participants who selfinjure would report more frequent dissociative experiences, higher rates of depression, higher levels of hopelessness, more frequent suicide attempts and higher levels of anxiety.

\section{METHODS}

\section{Participants}

The final sample was 44 participants - 11 who had engaged in NSSI at least once during their lifetime and 33 who had never engaged in NSSI. These participants were recruited from a larger sample of 883 students (see Procedure for more details). Participants were $81.8 \%$ female for the entire sample and for the NSSI group. The age range was 18 to 37 years, and the mean $( \pm$ SD) age was $20.25 \pm 4.30$ years. Participants were $56.8 \%$ freshman status, $29.5 \%$ sophomore status and $11.4 \%$ junior status. One participant declined to disclose her/his class rank. There was no statistical difference in age, academic year or sex between the NSSI and non-NSSI groups.

Forty-two participants self-identified as white or Caucasian, and two participants self-identified as multiracial. One hundred per cent of the NSSI group was white. The racial composition of the sample is typical for the mid-Atlantic university from which the sample was drawn. The West Virginia University (Morgantown, West Virginia, USA) institutional review board (IRB) approved the study. All participants were from undergraduate psychology classes.

\section{Measures}

Pain measure: An algometer pressure device was used as the measure of pain threshold and tolerance in the selected subset of participants (19). Originally developed by Forgione and Barber (20), the algometer used in the present study is based on work by Dougher et al (21), and described and diagrammed by Rainwater and McNeil (19). It has been used in previous research at West Virginia University $(22,23)$. The apparatus produces a slowly building and aching pain through the application of gradually increasing pressure to a portion of the participant's finger directly over the bone that is protected by little muscle or fat (ie, the second phalanx). Participants consecutively positioned the ring, middle and index fingers of their nondominant hand in the algometer device (in the same order for all participants). Each finger was placed between two pieces of wood and pressure was applied to the second phalanx of each finger via a dull lucite wedge attached to a wooden platform on which a $1750 \mathrm{~g}$ weight was placed. Each trial lasted a maximum of 5 min to avoid the possibility of tissue damage.

Measure 1 - pain threshold: Participants were instructed to indicate their pain threshold (ie, the point at which the pressure becomes painful) by touching a laminated, yellow yield sign placed next to his or her dominant hand. Pain threshold was measured according to the time interval between the start of the task and when he or she touched the yellow sign.

Measure 2-pain tolerance: Participants were instructed to indicate pain tolerance (ie, the point at which the pressure becomes too painful to continue) by touching a laminated, red stop sign placed on the table next to his or her dominant hand. Pain tolerance was measured according to the interval between the start of the task, and when he or she touched the red sign or until the 5 min maximum was reached.

Measure 3 - visual analogue scale: A visual analogue scale (VAS) is a line on which one end represents no pain and the other end represents extreme pain. The VAS is more sensitive than a simple verbal descriptive scale and has been useful in studies that compared pain severity between groups (24). The VAS was used to measure participants' self-reports of pain intensity (pain rating) on a continuum ranging from 0 (no pain sensation) to 100 (most intense pain sensation conceivable in the given situation). After each trial, participants were asked the following question: "On the scale from 0 to 100, how would you rate the worst pain you experienced during the task in the finger you had in the algometer pressure device?"

Screening measure: A 14-item self-report screening measure of sensation seeking was administered to the initial sample of 883 . The screening measure was developed by the authors of the study based on the Zuckerman Sensation Seeking Scale. The screening measure contained the first item of the Deliberate Self-Harm Inventory (DSHI): "I have intentionally (ie, on purpose) cut, carved or burned my wrists, arms or other areas of my body (without intending to kill myself)". Possible responses were never, one to four times, five to nine times, or 10 times or more (16). This screening item was used to determine whether participants met the criterion for inclusion in one of two 
groups: the NSSI group, who engaged in NSSI at least once during their lifetime, and the control group, who never engaged in self-injury.

Demographic and clinical history questionnaire: A 15-item self-report demographic questionnaire was used to obtain the age, sex, race/ethnicity and class rank of participants. In addition, the demographic and clinical history questionnaire was designed to determine whether participants met exclusionary criteria (eg, problems with the nondominant hand) and to obtain information about participants' history of suicide attempts and current mental health treatment (including currently prescribed medication).

Laboratory demographic questionnaire: The laboratory demographic questionnaire is an eight-item self-report questionnaire that was used to screen participants for inclusion in the algometer task on their arrival at the laboratory. Inclusion criteria included age of at least 18 years, the absence of heart problems, the absence of physical problems with the nondominant hand, duration of at least $12 \mathrm{~h}$ since the ingestion of any pain medication and the absence of Raynaud's disease. With the exception of age, inclusion in the algometer task was based on these variables because they have been found to affect pain tolerance and thresholds.

DSHI: The DSHI is a 17-item self-report measure designed to measure NSSI through items such as "Have you ever burned yourself with a cigarette? How old were you when you did this?" Gratz (25) found that the DSHI had sufficient test-retest reliability over a two- to four-week period (0.68) with regard to discriminating between participants who engaged in NSSI and those who did not. The DSHI was significantly correlated with other measures of self-harm. This measure was used to gather a more detailed history of participants' NSSI (eg, methods of self-injury, and first and most recent episode of self-injury).

DES: The DES is a 28-item self-report measure designed to assess the frequency and severity of dissociative experiences. Respondents are asked to indicate the frequency of such experiences using a 100-point scale. Test-retest reliability of the DES has yielded a correlation of 0.84 over a four- to eightweek interval. The convergent validity of the DES with other measures of dissociation was high (combined effect size $d=1.05$ ) (26).

Beck Depression Inventory - Second Edition: The Beck Depression Inventory (BDI) - Second Edition (BDI-II) is a 21-item self-report measure developed to measure the severity of depression. The BDI has good test-retest reliability over a one-week interval (alpha $=0.93)$. The convergent validity between the BDI-II and the previous version (the BDI-IA) is 0.93 (27).

Beck Hopelessness Scale: The Beck Hopelessness Scale (BHS) is a 20-item self-report measure developed to measure pessimism in adults. Items are rated on a four-point scale. Higher scores indicate greater hopelessness. The test-retest reliability of the BHS over one week is 0.69. The concurrent validity of clinical ratings of hopelessness and the BHS among a general medical sample was a correlation of 0.74 (28).

Anxiety Sensitivity Inventory: The Anxiety Sensitivity Inventory (ASI) is a 16-item self-report measure developed to assess fear of anxiety-related symptoms. Items are rated on a five-point scale. Test-retest reliability of the ASI is satisfactory with correlations ranging from 0.71 to 0.75 (29).

\section{Procedure}

Eight hundred eighty-three participants were administered all of the self-report measures via West Virginia University's online Sona system (Sona Systems, Estonia), a web-based survey management system for universities. The online availability of the measures allowed participants to participate in the study at their convenience. The measures were available online for seven months and participants completed them throughout that time period. The review of an online IRB-approved consent and a Health Insurance Portability and Accountability Act form was required to obtain access to the online measures. The consent form explained that participation on Sona is anonymous and that data are identified only by an identification number. Given the nature of the population and experimental question, a list of local mental health resources appeared as closing text when the self-report measures were complete.

Eligibility to participate in the study was based on participants' responses to the screening item on the screening measure. Participants who endorsed engaging in NSSI at least once during their lifetime and those who never engaged in NSSI were eligible to participate in the NSSI group and the control group, respectively. Students were invited to participate in the study by a Sona administrator via e-mail. The administrator was not informed of the purpose of the study. The e-mail invitation explained that students were eligible to participate in a study of pain perception based on their responses, but did not specify that they were selected based on whether they engaged in self-injury. The e-mail invitation described the location of the study, how long participants should expect the study to take $(30 \mathrm{~min})$, and stated that for their participation, participants would be entered into a raffle to receive one of four US $\$ 75$ prizes. The details of the study were not described to participants before their arrival. The basis for participant selection and the details of the study were intentionally not revealed to participants to decrease the likelihood of biasing their performance. Using the Sona system, invited participants signed up for a 30 min time slot during which they were to complete the algometer task. The experimenter was not informed of the group to which participants belonged.

Initially, the inclusion criterion for the NSSI group was to have engaged in NSSI 10 or more times (based on the criterion established in Gratz's study [25]). Because only 2.9\% of the respondents met that criterion, it was broadened to include participants with a lifetime history of NSSI to increase the sample size. One hundred forty-eight students reported engaging in NSSI at least once during their lifetime. All were invited to participate in the study. Only 11 students $(7.4 \%)$ participated.

Invitations were sent weekly, across seven months, to individuals eligible for both groups. Fifty-eight students agreed to participate in the study during that time. Eleven participants reported engaging in NSSI at least once during their lifetime. Forty-seven participants reported never engaging in self-injury. Although matching 11 of the 47 participants without a history of NSSI to the 11 with a history of NSSI would have yielded equal cell sizes, it would also have resulted in large SDs. To decrease the SDs and increase the likelihood of detecting a smaller effect size (decrease the likelihood of type 2 error), an unequal cell size was maintained. The larger group was trimmed to make the cell sizes less disparate. Thirty-three of the 
TABLE 1

Average pain tolerance, threshold and ratings by group

\begin{tabular}{lcccc}
\hline & Control $(\mathbf{n}=\mathbf{3 3})$ & Self-injury $(\mathbf{n}=\mathbf{1 1})$ & $\mathbf{F}(\mathbf{1}, \mathbf{4 2})$ & $\mathbf{d}^{\mathbf{2}}$ \\
\hline Threshold, $\mathrm{s}$ & $13.99 \pm 19.41$ & $41.30 \pm 87.95$ & 2.89 & 0.06 \\
Tolerance, $\mathrm{s}$ & $44.75 \pm 63.83$ & $109.18 \pm 127.02$ & $4.93^{\star}$ & 0.11 \\
Pain rating & $60.84 \pm 19.56$ & $46.51 \pm 21.45$ & $4.22^{\star}$ & 0.09 \\
\hline
\end{tabular}

Data presented as mean $\pm S D .{ }^{*} P<0.05$

47 participants were matched to the NSSI group based on age and sex to create a ratio of $3: 1$.

Experimenters were the primary investigator of the study and four research assistants. Three experimenters were women and two were men. Female and male experimenters administered the algometer task protocol to approximately the same number of female and male participants.

Experimenters were trained to administer the algometer task protocol from a printed script. Each experimenter administered the protocol without errors at least twice over a one-week interval. Experimenters also administered the protocol to at least one pilot participant before collecting data for the study. Experimenters always used the printed script when administering the protocol.

The algometer task took place in an office-sized conference room in the psychology department. Participants were shown the algometer and the task was briefly described to them. Participants then read the IRB-approved consent and Health Insurance Portability and Accountability Act forms. Participants were instructed on the algometer task and were asked to indicate that they understood the instructions before the task began. The task was terminated when the participant touched the red stop sign or after 5 min elapsed. The task was performed for the ring, index and middle fingers of each participant. Using the VAS, participants were asked to rate the pain they experienced after each trial (three separate times). After the termination of the algometer task, participants were debriefed regarding the purpose of the study.

\section{Data screening}

\section{RESULTS}

To exclude participants who may have responded randomly to the self-report measures, participants who completed the questionnaires in less than 10 min were not included in the study. Nineteen of the 883 participants' data were excluded for this reason. Four participants endorsed 'decline to answer' for all survey items and were also excluded from analyses.

\section{Rates of self-injury}

Among the larger sample of 860 participants, 144 (16.8\%) reported engaging in NSSI at least once during their lifetime. Ninety-six $(11.2 \%)$ participants reported engaging in NSSI one to four times, $23(2.7 \%)$ engaged in NSSI five to nine times and $25(2.9 \%)$ participants reported engaging in NSSI 10 or more times. Three participants declined to report whether they had engaged in self-injury.

Among the final sample of 44 participants, five (11.4\%) reported engaging in NSSI one to four times, three (6.8\%) engaged in NSSI five to nine times, and three $(6.8 \%)$ reported engaging in NSSI 10 or more times. All of the 11 participants reported engaging in NSSI within the past year. Four participants reported engaging in NSSI within the previous six months.
TABLE 2

Pain tolerance, pain threshold and pain rating by trial

\begin{tabular}{lcccc}
\hline & Control $(\mathbf{n}=\mathbf{3 3})$ & Self-injury $(\mathbf{n}=\mathbf{1 1})$ & $\mathbf{F}(\mathbf{1}, \mathbf{4 2})$ & $\mathbf{d}^{2}$ \\
\hline Threshold 1 & $11.76 \pm 14.34$ & $45.82 \pm 94.32$ & $4.21^{*}$ & 0.09 \\
Threshold 2 & $15.76 \pm 25.75$ & $37.91 \pm 87.19$ & 1.75 & 0.04 \\
Threshold 3 & $14.48 \pm 20.18$ & $37.73 \pm 87.59$ & 2.09 & 0.05 \\
Tolerance 1 & $37.76 \pm 53.20$ & $113.73 \pm 134.47$ & $7.37^{*}$ & 0.15 \\
Tolerance 2 & $49.97 \pm 77.61$ & $104.27 \pm 127.43$ & 2.88 & 0.06 \\
Tolerance 3 & $47.61 \pm 74.12$ & $102.18 \pm 128.99$ & 3.02 & 0.07 \\
Pain rating 1 & $58.55 \pm 21.11$ & $48.64 \pm 21.69$ & 1.80 & 0.04 \\
Pain rating 2 & $60.76 \pm 18.96$ & $49.55 \pm 26.78$ & 2.33 & 0.05 \\
Pain rating 3 & $63.24 \pm 21.77$ & $56.36 \pm 25.01$ & 0.77 & 0.02 \\
\hline
\end{tabular}

Data presented as mean $\pm S D$. 1 = ring finger, $2=$ middle finger and $3=$ index finger. Threshold and tolerance were measured in seconds. ${ }^{*} P<0.05$

\section{Methods of self-injury}

The most common methods of NSSI were cutting (72.7\%), severely scratching the skin to the extent that scarring or bleeding occurred (36.4\%), and sticking sharp objects into the skin, not including tattoos, ear piercing, body piercing or drug use $(27.3 \%)$.

\section{Pain tolerance and threshold}

Each participant's average pain threshold, pain tolerance and pain rating across all three trials were calculated and the mean scores for each group were compared using one-way ANOVA. Table 1 presents participants' average pain threshold, pain tolerance and pain rating by group across all three algometer trials. Separate ANOVAs compared pain threshold, pain tolerance and pain ratings between groups for each of the three trials. Analyses indicated that there were significant differences in the expected direction on two of the three variables: average pain tolerance and average pain rating. Participants in the control group indicated that the pressure produced by the algometer was too painful to continue after an average of $44.75 \pm 63.83 \mathrm{~s}$, while participants in the NSSI group did not indicate that the pressure from the algometer was too painful to continue until after an average of $109.18 \pm 127.02 \mathrm{~s}$ had elapsed. Participants in the control group rated the intensity of the pain they experienced during the algometer task an average of $60.84 \pm 19.56$ on a scale of 0 to 100 , while participants in the NSSI group provided a much less intense average rating of $46.51 \pm 21.45$.

To further explore differences between the two groups, the average pain threshold, pain tolerance and pain rating for each of the three trials were compared between the two groups using ANOVA. Table 2 presents participants' pain threshold, pain tolerance and pain rating by group for each algometer trial. Analyses revealed that there were significant differences in the expected direction for pain threshold and tolerance on the first of the three trials. During trial 1, participants in the control group indicated that the pressure from the algometer device had become painful after an average of only $11.76 \pm 14.34 \mathrm{~s}$. During that same trial, participants in the NSSI group did not indicate that the pressure from the algometer device had become painful until an average of $45.82 \pm 94.32 \mathrm{~s}$ had elapsed. Furthermore, participants in the control group indicated that the pressure produced on the ring finger by the algometer was too painful to continue after an average of $37.76 \pm 53.20 \mathrm{~s}$, while participants in the NSSI group were able to tolerate the pressure produced on the ring finger by the algometer for an 
TABLE 3

Psychological measures by group

\begin{tabular}{lcccc}
\hline Scale & Control $(\mathbf{n}=\mathbf{3 3})$ & Self-injury $(\mathbf{n}=\mathbf{1 1})$ & $\mathbf{F}(\mathbf{1}, \mathbf{4 2})$ & $\mathbf{d}^{\mathbf{2}}$ \\
\hline BDI-II & $9.03 \pm 8.32$ & $19.18 \pm 13.00$ & $9.15^{*}$ & 0.18 \\
BHS & $1.85 \pm 1.66$ & $5.09 \pm 4.66$ & $11.94^{*}$ & 0.22 \\
ASI & $18.24 \pm 7.29$ & $16.45 \pm 10.67$ & 0.39 & 0.01 \\
DES & $13.84 \pm 9.39$ & $16.01 \pm 14.34$ & 0.33 & 0.01 \\
\hline
\end{tabular}

Data presented as mean $\pm S D$. ${ }^{*} P<0.01$. ASI Anxiety Sensitivity Index; $B D I-I I$ Beck Depression Inventory - Second Edition; BHS Beck Hopelessness Scale; DES Dissociative Experiences Scale

average of $113.73 \pm 134.47 \mathrm{~s}$ before it became too painful to continue. The groups did not differ significantly in pain threshold and tolerance during trials 2 and 3 .

\section{Psychological measures}

To test hypothesis 2 , the mean scores for each group on the five psychological measures were compared using ANOVA. Table 3 presents participants' mean scores on the psychological measures by group. Results indicated that the groups differed significantly in the expected direction on two measures: the BDI-II and the BHS. The control group obtained an average score of $9.03 \pm 8.32$ on the BDI-II while the NSSI group scored an average of $19.18 \pm 13.00$. Similarly, the control group obtained an average score of $1.85 \pm 1.66$ on the BHS while the NSSI group scored an average of 5.09 \pm 4.66 . There were no significant differences in ASI or DES scores between the two groups.

To control for potential effects of the psychological variables on pain tolerance, pain threshold and pain rating, ANCOVAs were used to compare the two groups on average tolerance, average pain rating, and threshold and tolerance during the first trial while controlling for the effects of the psychological variables that were significantly different between the two groups.

Tables 4 and 5 present participants' adjusted mean scores on the significant pain threshold, pain tolerance and pain rating variables while covarying for scores on the two measures that were significantly different between the two groups: BDI-II and BHS. When controlling for scores with regard to the BDI-II, analyses indicated that although the difference in average tolerance and tolerance during the first trial was still significant, the average pain rating and threshold during the first trial were no longer significantly different. When controlling for scores on the BHS, there was still a significant difference between groups on all variables except threshold during the first trial. Thus, some of the variation in pain rating and pain threshold between the two groups was accounted for by psychological variables.

Two participants in the NSSI group reported a previous suicide attempt compared with zero in the non-NSSI group. The analyses confirmed the hypothesis that participants who self-injure would report more frequent suicide attempts $(\mathrm{F}[1,43]=7.00, \mathrm{P}=0.01)$.

\section{Post hoc analyses}

Post hoc analyses were conducted to examine potential differences among individuals who participated in the algometer task and those who were invited to participate, but did not. The analyses did not reveal any significant differences on the psychological measures between individuals who self-injured
TABLE 4

Pain tolerance, pain threshold and pain ratings covaried for the Beck Depression Inventory - Second Edition score

\begin{tabular}{|c|c|c|c|c|c|c|}
\hline & \multicolumn{2}{|c|}{ Control $(n=33)$} & \multicolumn{2}{|c|}{ Self-injury $(n=11)$} & \multirow[b]{2}{*}{$F(1,42)$} & \multirow[b]{2}{*}{$d^{2}$} \\
\hline & Adj $M$ & Std E & Adj M & Std E & & \\
\hline Tolerance & 42.98 & 15.03 & 114.49 & 27.34 & $4.90 *$ & 0.11 \\
\hline Threshold & 13.06 & 8.32 & 44.10 & 15.14 & 3.01 & 0.01 \\
\hline Pain rating & 60.71 & 3.62 & 46.91 & 6.59 & 3.15 & 0.07 \\
\hline Threshold 1 & 11.67 & 8.63 & 46.09 & 15.70 & 3.44 & 0.08 \\
\hline Tolerance 1 & 38.53 & 14.53 & 111.42 & 26.44 & $5.45^{\star}$ & 0.12 \\
\hline
\end{tabular}

Threshold and tolerance measured in seconds. ${ }^{*} P<0.05$. Threshold 1 is the threshold for trial 1 (ring finger); tolerance 1 is the tolerance for trial 1 (ring finger). Adj M Adjusted mean; Std E Standard error

\section{TABLE 5}

Pain tolerance, pain threshold and pain ratings covaried for the Beck Hopelessness Scale score

\begin{tabular}{|c|c|c|c|c|c|c|}
\hline & \multicolumn{2}{|c|}{ Control $(n=33)$} & \multicolumn{2}{|c|}{ Self-injury $(n=11)$} & \multirow[b]{2}{*}{$F(1,42)$} & \multirow[b]{2}{*}{$d^{2}$} \\
\hline & Adj $M$ & Std E & Adj M & Std E & & \\
\hline Tolerance & 41.78 & 15.09 & 118.13 & 27.81 & $5.34^{*}$ & 0.12 \\
\hline Threshold & 13.20 & 8.40 & 43.67 & 15.48 & 2.74 & 0.06 \\
\hline Pain rating & 61.35 & 3.64 & 44.99 & 6.71 & $4.22^{\star}$ & 0.09 \\
\hline Threshold 1 & 12.80 & 8.67 & 42.69 & 15.99 & 2.48 & 0.06 \\
\hline Tolerance 1 & 37.94 & 14.66 & 113.19 & 27.02 & $5.50^{\star}$ & 0.12 \\
\hline
\end{tabular}

Thresholds and tolerance measured in seconds. ${ }^{*} P<0.05$. Threshold 1 is the threshold for trial 1 (ring finger); tolerance 1 is the tolerance for trial 1 (ring finger). Adj M Adjusted mean; Std E Standard error

and participated in the algometer task, and those who did not participate.

\section{DISCUSSION}

\section{Major findings and implications}

As hypothesized, participants who reported engaging in NSSI had a significantly higher pain tolerance than participants who did not engage in self-injury. Similarly, participants who engaged in NSSI rated the pain as significantly less intense than participants who did not engage in self-injury. These findings are consistent with Joiner's theory (15) that greater experience with NSSI leads to an increased capacity for selfharm through desensitization to fear and pain by which it may be accompanied. However, given the cross-sectional study design, these results should not be interpreted as support for a causal or directional relation between NSSI and pain perception.

Interestingly, although there was a significant difference between the groups for threshold during the first trial (ring finger), there was no significant difference in average pain threshold. When pain threshold was averaged across the three trials, participants who self-injured perceived the pressure as painful just as quickly as participants who did not self-injure, but rated the pain as less intense overall and were able to tolerate the pain longer.

The absence of a difference in average pain threshold is inconsistent with the finding that individuals with BPD who self-injure have both higher pain tolerance and threshold than control participants, and may be interpreted several ways. It may suggest that the sample of community participants included in the NSSI group may not experience the same degree of pain insensitivity as a sample of individuals with BPD 
who self-injure. The nature of the algometer task may also explain the failure to observe a difference in average pain threshold between the two groups. Previous studies have demonstrated that individuals who self-injure may be particularly likely to experience reductions in pain perception when experiencing distress, which is the typical emotional state during an actual episode of NSSI $(12,30)$. The algometer task did not include a manipulation of the emotional state of the participants. Finally, the absence of this finding may be a result of inadequate power, particularly because there was a significant difference between the groups for threshold during the first trial.

The finding that groups differed in pain threshold and tolerance on trial 1, but not on trials 2 and 3 , raises questions about the possibility of habituation to the pressure of the algometer among both groups. Interestingly, after the first trial, the two groups performed similarly, with the self-injury group demonstrating lower tolerance and threshold, and the control group demonstrating generally higher tolerance and threshold. Furthermore, both groups' pain ratings actually increased across the three trials. Because the order of the fingers to which trials 1, 2 and 3 were administered was not counterbalanced, it is difficult to determine whether experience with the algometer or the order of the fingers influenced performance across the three trials.

Among this sample, participants who engaged in NSSI scored significantly higher on the BDI-II and BHS than participants who did not engage in NSSI. In fact, the self-injury group scored within the clinical range on the BDI-II, whereas the control group did not score within the clinical range. These findings are consistent with previous research (6). Analyses revealed that some of the differences in pain tolerance and pain ratings may have been influenced by psychological variables. When pain tolerance, pain threshold and pain rating were compared while controlling for depression, there was no longer a significant difference in average pain rating or pain threshold during the first trial between the groups. Thus, depression accounted for some of the variance in pain rating and pain threshold.

The current study did not replicate previous findings that individuals who self-injure have higher levels of anxiety and dissociation. The two groups did not significantly differ on ASI or DES scores. Failure to observe some of the hypothesized differences may have been due to the limited power from the small sample size. Among the statistically significant findings, effect sizes were relatively low. Alternatively, the absence of differences may suggest that the sample of community participants included in the NSSI group in the current study experienced less psychological distress than samples included in studies that found elevated anxiety and dissociation scores.

Finally, although all participants reported engaging in NSSI within the past year, only four reported engaging in NSSI within the previous six months. Ludäscher et al (31) found that individuals with BPD who reported NSSI within the previous six months demonstrated lower pain sensitivity than individuals with BPD who last engaged in NSSI more than six months previously. However, individuals with BPD who engaged in NSSI more than six months previously still had lower pain sensitivity than controls. Using six months as a definition for current NSSI, the sample in the current study is not representative of individuals with current NSSI. This may suggest that individuals with a history of NSSI, regardless of whether it is current, have less sensitivity to pain than individuals with no experience with NSSI.

\section{Limitations}

A primary limitation of the current study is the small sample size, which may have limited the power to detect differences between the groups. However, it should be noted that the sample of self-injurious participants examined in the present study $(\mathrm{n}=11)$ is comparable with the samples examined in previous research examining pain thresholds and tolerance among patients with BPD. Russ et al (17) examined pain perception among 11 participants with BPD who self-injured, Bohus et al (12) examined pain perception among 12 participants with BPD who self-injured, and Ludäscher et al (31) examined differences in pain perception between a group of 11 patients with BPD who no longer engaged in NSSI and 13 who did.

A second limitation relates to generalizability. First, undergraduates may be a relatively homogeneous group. Young adults in different social contexts may have responded differently. Second, the majority of individuals in the NSSI group reported relatively few episodes of NSSI. Therefore, the results may not be generalizable to individuals who report more frequent episodes of NSSI.

A third limitation relates to potentially confounding variables. Participants were not formally assessed for psychiatric disorders. Because decreased pain sensitivity is associated with a variety of mental health disorders $(9,32)$, it cannot be concluded that differences in pain perception were associated with NSSI and not some other disorder.

\section{Future directions}

The current study contributes to the literature examining pain perception among individuals who engage in self-injury. It supports findings that some individuals who self-injure may do so with an increased capacity to endure pain compared with individuals who do not self-injure (12). Extant literature $(16,17)$ suggests two significant clinical associations to diminished pain perception during self-injury: more frequent suicide attempts and an increased likelihood of using NSSI to alleviate negative affect. NSSI intended to alleviate negative affect is associated with increased risk for suicide compared with NSSI performed for other functions (4). Therefore, individuals who self-injure with an increased capacity to endure pain may be at increased risk for negative outcomes. Future studies should examine the relation among NSSI in the absence of pain, NSSI intended to alleviate negative affect, as well as suicide risk.

Given that some of the variance in pain rating and pain threshold was accounted for by scores on the BDI-II, future studies should examine potential mediating or moderating effects of depression on the relation between NSSI and pain perception. Furthermore, because the small sample size may have limited power to detect differences between the groups, future studies should replicate findings among larger, more diverse community samples, particularly adolescents, among whom the highest rates of NSSI have been reported (33). 
Specifically, the use of a longitudinal design could more adequately examine the hypothesis that experience with NSSI leads to decreased pain sensitivity. Finally, based on the current study, it is unclear whether experience with the algometer caused habituation across trials. Future studies should examine this hypothesis. Similar or differential habituation during a laboratory task among individuals who do and do not engage in NSSI would shed light on whether

\section{REFERENCES}

1. Klonsky ED. Non-suicidal self-injury: An introduction. J Clin Psychol 2007;63:1039-43.

2. Gratz KL. Risk factors for deliberate self-harm among female college students: The role and interaction of childhood maltreatment, emotional inexpressivity, and affect intensity/reactivity. Am J Orthopsychiatry 2006;76:238-50.

3. Nock M, Prinstein M. A functional approach to the assessment of self-mutilative behavior. J Consult Clin Psychol 2004;72:885-90.

4. Nock M, Prinstein M. Contextual features and behavioral functions of self-mutilation among adolescents. J Abnorm Psychol 2005;114:140-6.

5. Briere J, Gil E. Self mutilation in clinical and general population samples: Prevalence, correlates and functions. Am J Orthopsychiatry 1998;68:609-20.

6. Ross S, Heath N. A study of the frequency of self-mutilation in a community sample of adolescents. J Youth Adolesc 2002;31:67-77.

7. Gratz KL, Conrad SD, Roemer L. Risk factors for deliberate self harm among college students. Am J Orthopsychiatry 2002;72:128-40.

8. Whitlock J, Eckenrode J, Silverman D. Self-injurious behaviors in a college population. Pediatrics 2006;117:1939-48.

9. Haw C, Hawton K, Houston K, Townsend E. Psychiatric and personality disorders in deliberate self-harm patients. Br J Psychiatry 2001;178:48-54.

10. Guertin T, Lloyd-Richardson E, Spirito A, Donaldson D, Boergers J. Self-mutilative behavior in adolescents who attempt suicide by overdose. J Am Acad Child Adolesc Psychiatry 2001;40:1062-9.

11. Klonsky ED, Oltmanns TF, Turkheimer E. Deliberate self-harm in a nonclinical population: Prevalence and psychological correlates. Am J Psychiatry 2003;160:1501-8.

12. Bohus M, Limberger M, Ebner U, et al. Pain perception during self-reported distress and calmness in patients with borderline personality disorder and self-mutilating behavior. Psychiatry Res 2000;95:251-60.

13. Russ MJ, Campbell SS, Kakuma T, Harrison K, Zanine E. EEG theta activity and pain insensitivity in self-injurious borderline patients. Psychiatry Res 1999;89:201-14.

14. Kemperman I, Russ M, Shearin E. Self-injurious behavior and mood regulation in borderline patients. J Pers Disord 1997;11:146-57.

15. Joiner T. Why People Die by Suicide. Cambridge, MA: Harvard University Press, 2005.

16. Nock M, Joiner T, Gordon K, Prinstein M, Lloyd-Richardson E. Non-suicidal self-injury among adolescents: Diagnostic correlates and relation to suicide attempts. Psychiatry Res 2006;144:65-72. individuals who engage in NSSI seem to have lower pain sensitivity through a unique or common mechanism.

DISCLOSURE: This article is based in part on the master's thesis of the first author under the direction of the second and third author. Portions of it were presented at the annual meeting of the Eastern Psychological Association (March 2008, Baltimore, Maryland, USA).
17. Russ MJ, Roth SD, Lerman A, et al. Pain perception in self-injurious patients with borderline personality disorder. Biol Psychiatry 1992;32:501-11.

18. Glenn CR, Klonsky DE. Social context during non-suicidal self-injury indicates suicide risk. Pers Individ Dif 2009;46:25-9.

19. Rainwater A, McNeil D. Reinventing the algometer: Synopsis of the literature and presentation of a reliable, inexpensive model. Behav Res Methods Instrum Comput 1991;23:486-92.

20. Forgione AG, Barber TX. A strain gauge pain stimulator. Psychophysiology 1971;8:102-6.

21. Dougher MJ, Goldstein D, Leight KA. Induced anxiety and pain. J Anxiety Disord 1987;1:259-64.

22. Vowles KE, McNeil DW, Sorrell JT, Lawrence SM. Fear and pain: Investigating the interaction between aversive states. J Abnorm Psychol 2006;115:821-33.

23. Kyle B, McNeil DW, Weinstein B, Mark J. Interaction of intensity and order of painful events. J Behav Med 2009;32:360-70.

24. Huskisson EC. Visual Analog Scales. In: Melzock R, ed. Pain Measurement and Assessment. New York: Raven Press, 1983:33-6.

25. Gratz KL. Measurement of deliberate self-harm: Preliminary data on the Deliberate Self-Harm Inventory. J Psychopathol Behav Assess 2001;23:253-63.

26. Carlson EB, Putnam FW. An update on the Dissociative Experiences Scale. Dissociation 1993;6:16-27.

27. Beck A, Steer R, Brown G. Manual for Beck Depression Inventory - II. San Antonio: The Psychological Corporation, 1996.

28. Beck A, Steer R. Manual for Beck Hopelessness Scale. San Antonio: The Psychological Corporation, 1993.

29. Peterson RA, Reiss S. Anxiety Sensitivity Index Revised test manual. Worthington: IDS Publishing Corporation, 1993.

30. Klonsky ED. The functions of deliberate self-injury: A review of the evidence. Clin Psychol Rev 2007;27:226-39.

31. Ludäscher P, Greffrath W, Schmahl C, et al. A cross sectional investigation of discontinuation of self-injury and normalizing pain perception in patients with borderline personality disorder. Acta Psychiatr Scand 2009;120:62-70.

32. Raymond N, Faris P, Thuras P, et al. Elevated pain threshold in anorexia nervosa subjects. Biol Psychiatry 1999;45:1389-92.

33. Lloyd-Richardson EE, Perrine N, Dierker L, Kelley M. Characteristics and functions of non-suicidal self-injury in a community sample of adolescents. Psychol Med 2007;37:1183-92. 


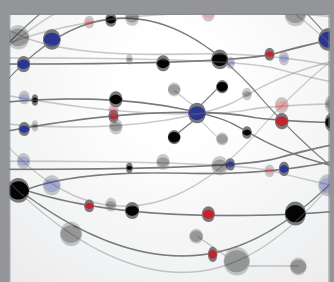

The Scientific World Journal
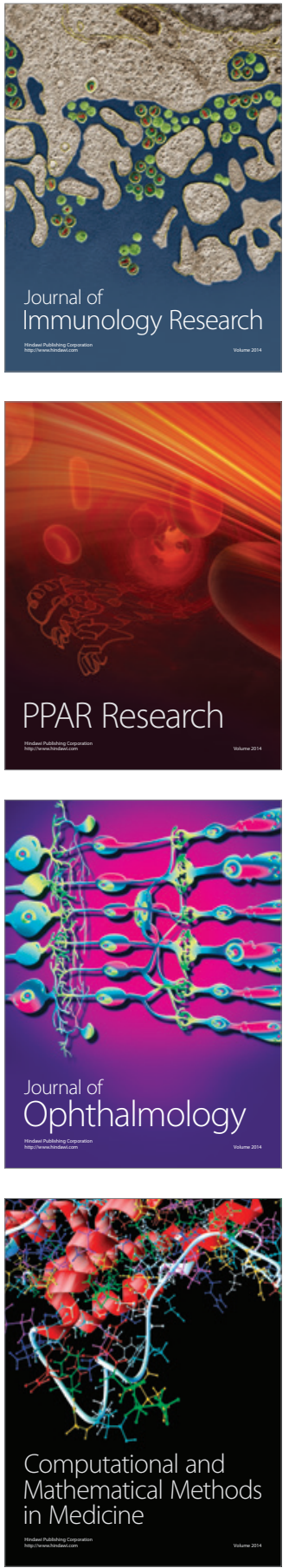

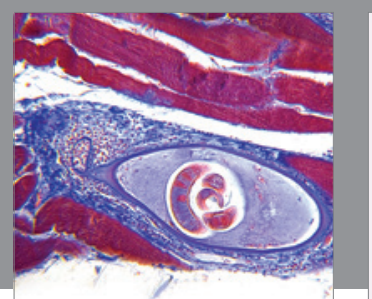

Gastroenterology Research and Practice

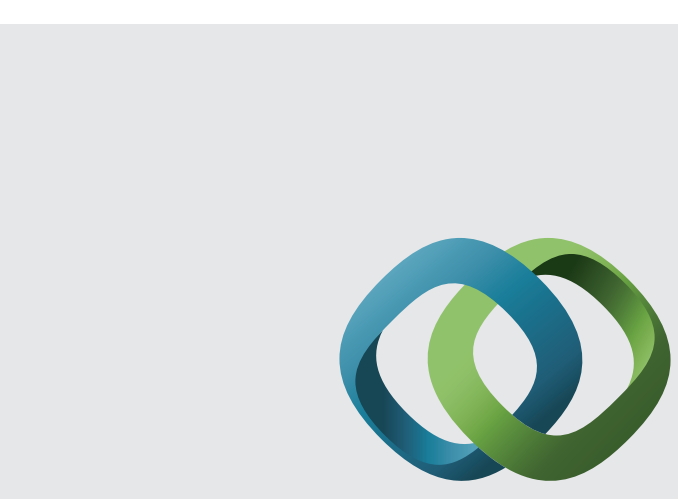

\section{Hindawi}

Submit your manuscripts at

http://www.hindawi.com
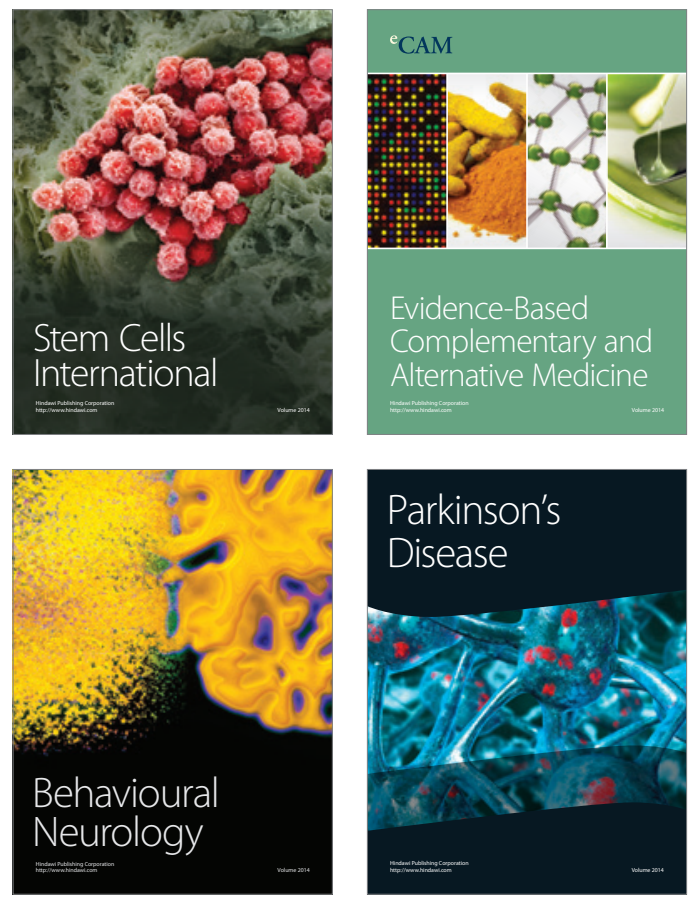
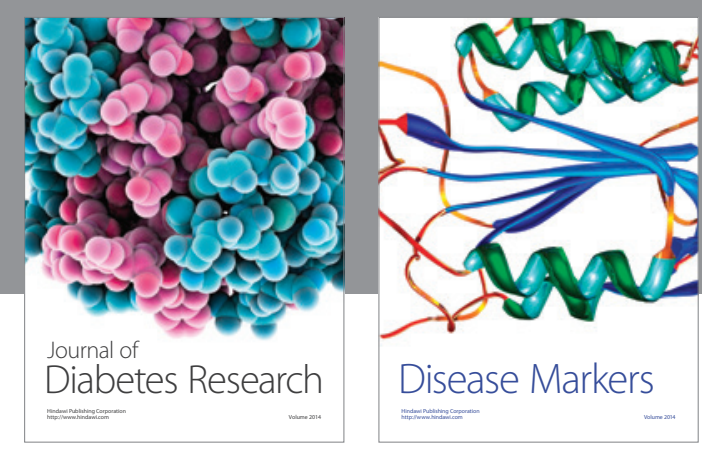

Disease Markers
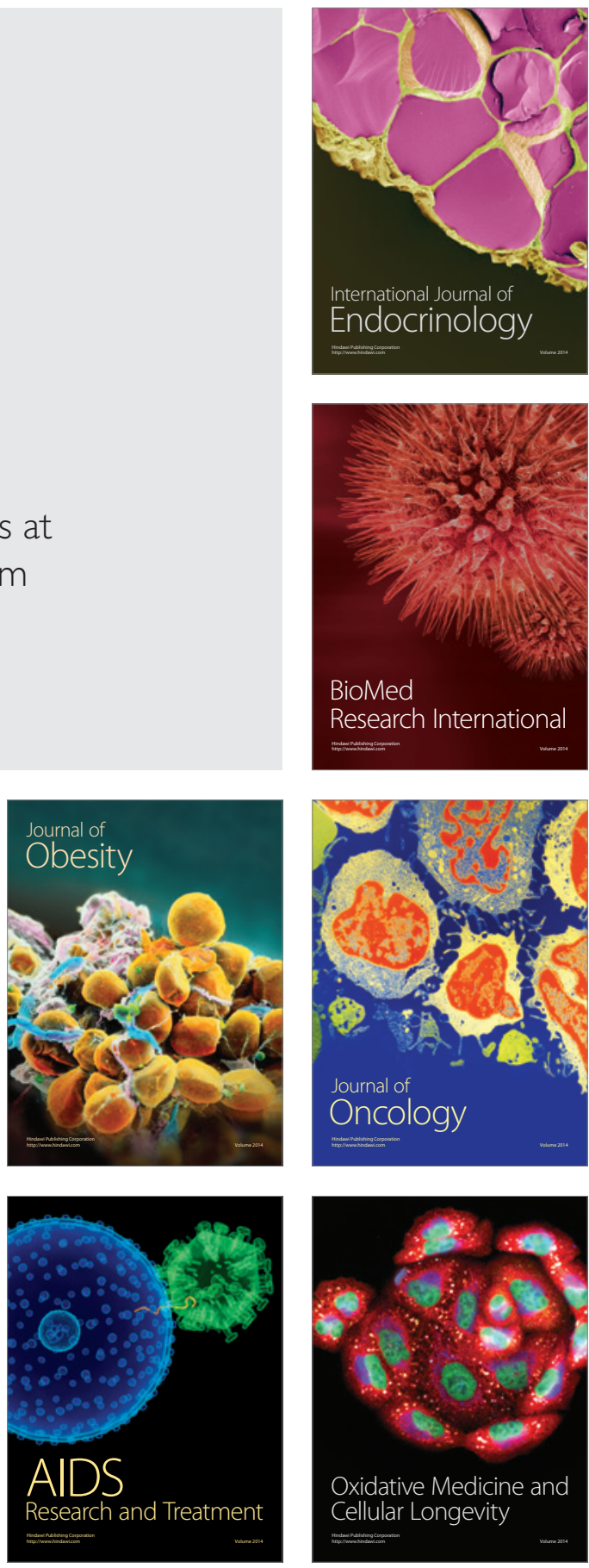